TRANSACTIONS OF THE

AMERICAN MATHEMATICAL SOCIETY

Volume 353, Number 2, Pages 609-634

S 0002-9947(00)02739-2

Article electronically published on October 23, 2000

\title{
ASYMPTOTIC CONVERGENCE OF THE STEFAN PROBLEM TO HELE-SHAW
}

\author{
FERNANDO QUIRÓS AND JUAN LUIS VÁZQUEZ
}

\begin{abstract}
We discuss the asymptotic behaviour of weak solutions to the Hele-Shaw and one-phase Stefan problems in exterior domains. We prove that, if the space dimension is greater than one, the asymptotic behaviour is given in both cases by the solution of the Dirichlet exterior problem for the Laplacian in the interior of the positivity set and by a singular, radial and self-similar solution of the Hele-Shaw flow near the free boundary. We also show that the free boundary approaches a sphere as $t \rightarrow \infty$, and give the precise asymptotic growth rate for the radius.
\end{abstract}

\section{INTRODUCTION}

A Stefan problem is a mathematical model for describing the melting of a body of ice in contact with a region of water. In one-phase Stefan problems, the temperature of the ice is supposed to be maintained at $0^{\circ} \mathrm{C}$. Hence, the unknowns are the temperature distribution in the water and the shape of the interface (free boundary) between ice and water, see $[\mathrm{Ru}],[\mathrm{Me}$. This problem leads to a mathematical model posed in one, two or three space dimensions. The mathematical problem is naturally generalized to arbitrary integer space dimension $n \geq 1$ and is still called the Stefan problem.

The Hele-Shaw problem is a two-dimensional mathematical model describing the movement of a viscous fluid confined in a narrow cell between two parallel plates, $[\mathrm{ST}]$, EJ]. It has applications in the plastics industry (injection moulding, [ $[\mathrm{R}]$ ), and in electromachining, $\mathrm{MGR}$. It can also be considered as the zero specific heat limit of the Stefan problem $[\mathrm{L},[\mathrm{LR}$, , being in that sense a simplified version of that famous problem. We will consider the natural generalization of this mathematical formulation in all space dimensions $n \geq 1$.

The aim of the present paper is to study the large-time behaviour of the solution to the initial-and-boundary-value problem in an exterior domain $\Omega$ for both models, and to show their relationship. We prescribe Dirichlet boundary data $g(x)$ on the compact set $\partial \Omega$, which are non-negative, non-trivial $(g \not \equiv 0)$ and constant in time. For the Stefan problem we also specify initial data

$$
\theta(x, 0)=\theta_{0}(x),
$$

which are assumed to be nonnegative and compactly supported in $\Omega$. For the Hele-Shaw problem we need only specify the initial support, which is bounded.

Received by the editors December 31, 1996 and, in revised form, October 28, 1997.

2000 Mathematics Subject Classification. Primary 35B40, 35R35.

Key words and phrases. Stefan problem, Hele-Shaw, asymptotic behaviour. 
We will show that there is a marked difference in the asymptotic behaviour of these problems in one and several dimensions. Thus, it is well known that in one space dimension there is convergence to a stationary state in the $x / t^{1 / 2}$-scale for both problems, but the asymptotic profile is not the same. On the contrary, we will show that in dimensions $n \geq 2$ the Stefan problem simplifies into the Hele-Shaw problem. Let us describe the results in some more detail:

1-D Hele-Shaw problem. To find $p(x, t)$ and $r(t)$ such that

$$
\begin{cases}\frac{\partial^{2} p}{\partial x^{2}}=0, & 0<x<r(t), t>0 \\ p(r(t), t)=0, & t \geq 0, \\ \frac{\partial p}{\partial x}(r(t), t)=-L r^{\prime}(t), & t>0 \\ p(0, t)=A, & t \geq 0 \\ r(0)=b . & \end{cases}
$$

In this case the problem can be solved explicitly, and the solution is

$$
p(x, t)=A\left[1-\frac{x}{\sqrt{b^{2}+\frac{2 A}{L} t}}\right]_{+} .
$$

It is immediate that the asymptotic behaviour is given by the self-similar solution

$$
p(x, t)=A\left[1-\frac{\zeta}{\sqrt{2 A / L}}\right]_{+}, \quad \zeta=x / \sqrt{t},
$$

with free boundary

$$
r(t)=(2 A / L)^{1 / 2} t^{1 / 2}
$$

1-D Stefan problem. To find $\theta(x, t)$ and $r(t)$ such that

$$
\begin{cases}c \frac{\partial \theta}{\partial t}=\frac{\partial^{2} \theta}{\partial x^{2}}, & 0<x<r(t), t>0, \\ \theta(x, 0)=\theta_{0}(x), & 0 \leq x \leq b, \\ \theta(r(t), t)=0, & t \geq 0, \\ \frac{\partial \theta}{\partial x}(r(t), t)=-L r^{\prime}(t), & t>0, \\ \theta(0, t)=A, & t \geq 0, \\ r(0)=b . & \end{cases}
$$

The constant $c$ represents the specific heat. It is well known that in this case the asymptotic behaviour of the solution is given by a self-similar solution of the form

$$
\theta(x, t)=A\left[1-\frac{E\left(c^{1 / 2} \zeta\right)}{E\left(\eta_{0}\right)}\right]_{+}, \quad \zeta=x / \sqrt{t},
$$

where $E(\cdot)$ is the error function and $\eta_{0}$ is the unique solution of the equation

$$
\mathcal{F}\left(\eta_{0}\right) \equiv \frac{\sqrt{\pi}}{2} \eta_{0} e^{\eta_{0}^{2} / 4} E\left(\eta_{0}\right)=\frac{A c}{L}
$$

The free boundary of the self-similar solution (1.2) is given by

$$
r(t)=\frac{\eta_{0}}{\sqrt{c}} t^{1 / 2}
$$


Although both functions $p$ and $\theta$ stabilize to the same constant level $A$ as $t \rightarrow \infty$ for every fixed $x>0$, the convergence is not uniform and the profiles giving the intermediate asymptotic behaviour are different. Besides, the growth rate of the free boundary is in both cases $t^{1 / 2}$, but the constants are not the same. However, the two problems are closely related. Indeed, it is well known that solutions of the Stefan problem converge to solutions of Hele-Shaw as $c \rightarrow 0$. We can in fact check that $\mathcal{F}^{-1}(S) \sim \sqrt{2 S}$ as $S \rightarrow 0$. Therefore, $\zeta_{0}=\eta_{0} / \sqrt{c} \rightarrow(2 A / L)^{1 / 2}$ as $c \rightarrow 0$. Moreover, the profile for the Stefan problem given in (1.2) converges to the profile for the Hele-Shaw problem appearing in (1.1).

The situation is different for $n>1$. Indeed, there is no self-similar solution which explains simultaneously the large-time behaviour of the solutions and interfaces of our problems. The actual behaviour can be described using the ideas of matched asymptotic expansions. The positivity set expands to cover in finite time any compact subset of the exterior domain, $\Omega$, and the solutions of both problems stabilize in the usual space coordinates to the solution of the exterior Dirichlet problem for the Laplacian. This is what we call the near field limit. On the other hand, we prove that, after a scaling of the form

$$
\begin{array}{lll}
y=x / t^{1 / n}, & v=u t^{(n-2) / n} & \text { if } n \geq 3, \\
y=x / \mathcal{R}(t), & v=u \log \mathcal{R}(t) & \text { if } n=2,
\end{array}
$$

where $\mathcal{R}(t) \sim C(t / \log t)^{1 / 2}$ as $t \rightarrow \infty$, and $u$ represents $\theta$ if we are studying the Stefan problem and $p$ if we are considering the Hele-Shaw flow, we have convergence in both problems to the same free boundary problem, which corresponds to a singular, radial, self-similar solution of the Hele-Shaw problem. This is what we call the far field limit. This means that in the far region, close to the free boundary, Hele-Shaw represents the reduced problem for Stefan (in the sense of Prandtl's theory of boundary layers). In practice, the heat equation of the Stefan problem loses the inertial term $\theta_{t}$ as $t \rightarrow \infty$ because this term becomes lower order with respect to the main terms at the asymptotic level. It is thus a case of asymptotic simplification, a rather usual phenomenon in nonlinear parabolic equations (see the survey $[\mathrm{V}]$ ). The analysis gives in particular the position of the free boundary as

$$
\begin{array}{ll}
|x| \sim C t^{1 / n} & \text { if } n \geq 3, \\
|x| \sim C(t / \log t)^{1 / 2} & \text { if } n=2
\end{array}
$$

for a precise constant $C>0$ which depends on the data. The matching between the two regions amounts to determining this constant in the far region.

A previous study of the asymptotic behaviour for the Stefan problem for $n \geq 3$ was done by Matano $[\mathrm{M}]$. He proved that any weak solution becomes eventually classical, that is, the interface between the ice and the water regions is sufficiently smooth for all large $t$. He also proved that the shape of the free boundary (i.e., the interface) tends to a sphere of radius $(C t)^{1 / n}$ as $t \rightarrow \infty$, and he calculated the constant. We establish the asymptotic developments for the solutions and interfaces for all $n \geq 2$. We also prove that the shape of the free boundary of the Hele-Shaw problem approaches a sphere, with precisely the same radius.

In conclusion, we show that there is a strong difference between the one-dimensional and the $n \geq 2$ cases: for $n=1$ solutions of the Stefan problem do not eventually become solutions of Hele-Shaw, while for $n \geq 2$ they do. Extending the analysis for radial solutions of Section 3 to non-integer dimensions (which is formally possible) shows that $n=2$ is precisely the critical value which separates 
the interface behaviours of the form $|x| \sim C t^{1 / n}$, valid for $n>2$, from $|x| \sim C t^{1 / 2}$, valid for $n<2$. The log term in the interface expression for $n=2$ is a visual proof of such critical behaviour. Returning to our problem in integer dimensions, there is an explicit self-similar solution giving the asymptotic behaviour of Stefan when $n=1$ which is not a solution of Hele-Shaw. On the other hand, for $n \geq 2$ we need two matched developments to explain the asymptotic behaviour of the solutions in the two problems. For the record, the development in the region close to the free boundary is always self-similar. The influence of the compactly supported initial data disappears (to first order) in all cases.

\section{Preliminaries}

In order to state the above results more precisely we introduce some notation. We assume that we are given a bounded domain $G_{0} \subset \mathbb{R}^{n}$ whose boundary consists of two smooth connected hypersurfaces $\Gamma$ and $\Gamma_{0}$, where $\Gamma$ lies inside $\Gamma_{0}$ and bounds a simply connected domain $G$, i.e., $\partial G=\Gamma$. We will assume without loss of generality that $0 \in G$.

Classical formulation of the Hele-Shaw problem. To find functions $s(x) \geq 0$ and $p(x, t) \geq 0$, defined for $t>0, x \in \mathbb{R}^{n} \backslash G$, which satisfy

$$
\begin{cases}\Delta p=0 & \text { in }\left\{(x, t): x \in \mathbb{R}^{n} \backslash \bar{G}, t>s(x)\right\}, \\ p=0 & \text { if } t=s(x), x \in \mathbb{R}^{n} \backslash\left(G_{0} \cup \bar{G}\right), \\ \nabla p \cdot \nabla s=-L & \text { if } t=s(x), x \in \mathbb{R}^{n} \backslash\left(G_{0} \cup \bar{G}\right), \\ p(x, t)=g(x) & \text { if } x \in \Gamma, t>0, \\ s(x)=0 & \text { if } x \in G_{0},\end{cases}
$$

where $L>0$ is a constant and $g$ is a function that is non-negative, non-trivial and continuous on $\Gamma$.

When this problem is used as a two-dimensional model to describe the movement of a viscous fluid between two parallel plates, the function $p$ is interpreted as the pressure in the fluid, the surface $t=s(x)$ is the interface between the fluid and the surrounding air, and the air pressure is considered to be identically 0 . At time $t$ the fluid occupies the subset $\Omega(t)$ of $\mathbb{R}^{2} \backslash G$ defined by $\Omega(t) \equiv\left\{x \in \mathbb{R}^{2} \backslash G: s(x)<t\right\}$.

Classical formulation of the Stefan problem. Solving this problem consists in finding functions $s(x) \geq 0$ and $\theta(x, t) \geq 0$, defined for $t \geq 0, x \in \mathbb{R}^{n} \backslash G$, which satisfy

$$
\begin{cases}\frac{\partial \theta}{\partial t}=\Delta \theta & \text { in }\left\{(x, t): x \in \mathbb{R}^{n} \backslash \bar{G}, t>s(x)\right\}, \\ \theta=0 & \text { if } t=s(x), x \in \mathbb{R}^{n} \backslash\left(G_{0} \cup \bar{G}\right), \\ \nabla \theta \cdot \nabla s=-L & \text { if } t=s(x), x \in \mathbb{R}^{n} \backslash\left(G_{0} \cup \bar{G}\right), \\ \theta(x, 0)=h(x) & \text { if } x \in G_{0}, \\ \theta(x, t)=g(x) & \text { if } x \in \Gamma, t>0, \\ s(x)=0 & \text { if } x \in G_{0},\end{cases}
$$

where $L>0$ is a constant (the latent heat), and $g, h$ are given functions satisfying:

(i) $g$ is non-negative, non-trivial and continuous on $\Gamma$;

(ii) $h$ is continuous in $\mathbb{R}^{n} \backslash G, h(x)>0$ if $x \in G_{0}, h(x)=0$ if $x \in \mathbb{R}^{n} \backslash\left(G_{0} \cup \bar{G}\right)$;

(iii) $h=g$ on $\Gamma$. 
In the application the function $\theta$ is interpreted as the temperature of the water, the surface $t=s(x)$ is the interface between water and ice, and the ice temperature is considered to be identically 0 . At time $t$ the water occupies the subset of $\mathbb{R}^{3} \backslash G$ defined by $\Omega(t) \equiv\left\{x \in \mathbb{R}^{3} \backslash G: s(x)<t\right\}$.

Remark. In both problems it is possible to take boundary data $g(x, t)$ depending on time. However, we study the asymptotic behaviour only for data that are constant in time.

The Hele-Shaw and Stefan problems do not necessarily have a global classical solution in the case of multi-dimensional spaces - once more in contrast with the one-dimensional case - as cusp-like singularities sometimes appear on the free boundary. However, they always have a weak solution that exists globally in time.

Weak formulation of the Hele-Shaw problem. Elliott and Janovský [EJ] introduced into the Hele-Shaw problem the Baiocchi transform $u(x, t)$, defined in $\left(\mathbb{R}^{n} \backslash \bar{G}\right) \times \mathbb{R}^{+}$as

$$
u(x, t)= \begin{cases}\int_{s(x)}^{t} p(x, \tau) d \tau & \text { if } x \in \Omega(t), \\ 0 & \text { otherwise. }\end{cases}
$$

For every $t>0$ the function $u(\cdot, t)$ satisfies

$$
\begin{cases}\Delta u=L & \text { in } \Omega(t) \backslash G_{0}, \\ \Delta u=0 & \text { otherwise. }\end{cases}
$$

Moreover, it satisfies the boundary conditions

$$
u=\frac{\partial u}{\partial n}=0 \quad \text { on } \Gamma(t) \equiv\left\{x \in \mathbb{R}^{n} \backslash\left(G_{0} \cup \bar{G}\right), t=s(x)\right\} .
$$

This variable satisfies an integrated version of the condition at the fixed boundary,

$$
u(x, t)=\int_{0}^{t} g(x, \tau) d \tau \quad \text { on } \Gamma .
$$

Hence $u$ is a solution of the nonlinear elliptic problem

$$
\begin{cases}\Delta u=\chi(x) H(u) & \text { in } \mathbb{R}^{n} \backslash \bar{G}, \\ u(x, t)=\int_{0}^{t} g(x, \tau) d \tau & \text { on } \Gamma .\end{cases}
$$

Here $\chi(x)=0$ in $G_{0}$ and $\chi(x)=L$ elsewhere, $H(u)=0$ for $u \leq 0, H(u)=1$ for $u>0$. Alternatively, $u$ can be regarded as the solution of a variational inequality, since

$$
u \geq 0, \chi(x)-\Delta u \geq 0 \text { and }(\chi(x)-\Delta u) u=0 .
$$

By using this formulation Elliott and Janovský were able to prove that there is a unique solution to (WHS). Given a solution $u$, we put

$$
\begin{aligned}
& \Omega(t)=\left\{x \in \mathbb{R}^{n} \backslash G: u(x, t)>0\right\}, \\
& \Gamma(t)=\partial \Omega(t) \backslash \partial G, \\
& p(x, t)= \begin{cases}\frac{\partial u}{\partial t}(x, t) & \text { if } x \in \Omega(t), \\
0 & \text { if } x \in \mathbb{R}^{n} \backslash G, x \notin \Omega(t) .\end{cases}
\end{aligned}
$$

The pair $(p, \Gamma(t))$ is known as the weak solution to the Hele-Shaw problem (WHS). 
We will use the following comparison result from [KLV]:

Proposition 2.1. Suppose that $\chi \geq \hat{\chi}$ and $g \leq \hat{g}$. Let $u$, $\hat{u}$ be the solutions to problem (WHS) for $\chi, g$ and $\hat{\chi}, \hat{g}$ respectively. Then

$$
u \leq \hat{u} .
$$

Remark. Under the same conditions it is easily proved with the same method that was suggested for the Stefan problem by Friedman and Kinderlehrer (see the remark after Lemma 2.5 in [FK]) that

$$
p \equiv \frac{\partial u}{\partial t} \leq \frac{\partial \hat{u}}{\partial t} \equiv \hat{p}
$$

Weak formulation of the Stefan problem. In this formulation, due to Duvaut D, $\theta(x, t)$ is given in the form $\theta=u_{t}$, where $u$ is the solution to a certain variational inequality. More precisely, let $u(x, t)$ be defined by

$$
u(x, t)= \begin{cases}\int_{s(x)}^{t} \theta(x, \tau) d \tau & \text { if } x \in \Omega(t), \\ 0 & \text { otherwise. }\end{cases}
$$

If we compute the differential equation satisfied by $u$, we obtain

$$
\left\{\begin{array}{l}
\frac{\partial u}{\partial t}-\Delta u=-L, \quad x \in \mathbb{R}^{n} \backslash\left(G_{0} \cup \bar{G}\right), s(x)<t, \\
\frac{\partial u}{\partial t}-\Delta u=h(x), \quad x \in G_{0}, s(x)<t .
\end{array}\right.
$$

Moreover, it satisfies the boundary conditions

$$
u=\frac{\partial u}{\partial n}=0 \quad \text { if } t=s(x), x \in \mathbb{R}^{n} \backslash\left(G_{0} \cup \bar{G}\right) .
$$

This variable also satisfies an integrated version of the condition imposed at the fixed boundary:

$$
u(x, t)=\int_{0}^{t} g(x, \tau) d \tau \quad \text { on } \Gamma .
$$

Hence $u$ satisfies the nonlinear parabolic problem

$$
\begin{cases}\frac{\partial u}{\partial t}-\Delta u=f(x) H(u) & \text { in }\left(\mathbb{R}^{n} \backslash \bar{G}\right) \times[0, \infty), \\ u(x, t)=\int_{0}^{t} g(x, \tau) d \tau & \text { on } \Gamma\end{cases}
$$

where

$$
f= \begin{cases}h(x) & \text { if } x \in G_{0}, \\ -L & \text { if } x \in \mathbb{R}^{n} \backslash\left(G_{0} \cup \bar{G}\right) .\end{cases}
$$

Alternatively, $u$ can be regarded as the solution of a variational inequality, since

$$
u \geq 0, \frac{\partial u}{\partial t}-\Delta u-f \geq 0 \text { and }\left(\frac{\partial u}{\partial t}-\Delta u-f\right) u=0 .
$$


By using this formulation Friedman and Kinderlehrer [FK] were able to prove that there is a unique solution to (WS). Given a solution $u$, we put

$$
\begin{aligned}
& \Omega(t)=\left\{x \in \mathbb{R}^{n} \backslash G: u(x, t)>0\right\}, \\
& \Gamma(t)=\partial \Omega(t) \backslash \partial G, \\
& \theta(x, t)= \begin{cases}\frac{\partial u}{\partial t}(x, t) & \text { if } x \in \Omega(t), \\
0 & \text { if } x \in \mathbb{R}^{n} \backslash G, x \notin \Omega(t) .\end{cases}
\end{aligned}
$$

The pair $(\theta, \Gamma(t))$ is known as the weak solution to the Stefan problem (WS). Properties of the solution can be found for instance in [FK], [L]. We will use the following comparison result from $\mathrm{FK}$ :

Proposition 2.2. Suppose that $f \leq \hat{f}$ and $g \leq \hat{g}$. Let $u$, $\hat{u}$ be the solutions to problem (WS) for $f, g$ and $\hat{f}, \hat{g}$ respectively. Then

$$
u \leq \hat{u}
$$

Moreover,

$$
\theta \equiv \frac{\partial u}{\partial t} \leq \frac{\partial \hat{u}}{\partial t} \equiv \hat{\theta}
$$

Caffarelli and Friedman $\mathrm{CF}$ have proved that $\theta$ is continuous in $\left(\mathbb{R}^{n} \backslash G\right) \times[0, \infty)$. It is not difficult to see that $\theta$ is smooth in the interior of the water region, since it satisfies there the heat equation in the sense of distributions. Further regularity of $\theta$ near the free boundary $\Gamma(t)$ depends on the regularity of $\Gamma(t)$. In other words, a weak solution is classical so long as $\Gamma(t)$ has no singular point. Indeed, any singular point appearing on the free boundary should be a cusp-like singularity, $[\mathrm{C}$, and the solution is $C^{\infty}$ inside the water region and up to the free boundary provided that this free boundary forms locally a $C^{1}$ hypersurface, $[\mathrm{KN}]$. It is also known that the free boundary is $C^{\infty}$ if it is $C^{1}$ or even simply Lipschitz, [KN], $\left.\mathrm{C}\right]$. To state these results more precisely we recall the notion of Lebesgue density.

Definition 2.3. Let $A \subset \mathbb{R}^{n}$ be a measurable set and let $x_{0}$ be any point in $\mathbb{R}^{n}$. We say $x_{0}$ has positive Lebesgue density with respect to $A$ if

$$
\liminf _{\rho \downarrow 0} \frac{\mu\left(A \cap B_{\rho}\left(x_{0}\right)\right)}{\mu\left(B_{\rho}\left(x_{0}\right)\right)}>0,
$$

where $\mu$ is the Lebesgue measure and $B_{\rho}\left(x_{0}\right)=\left\{x \in \mathbb{R}^{n}:\left|x-x_{0}\right|<\rho\right\}$.

Combining the $C^{\infty}$ smoothness criterion of Kinderlehrer and Nirenberg $[\mathrm{KN}$ and the $C^{1}$ smoothness criterion of Caffarelli [C], we get the following proposition:

Proposition 2.4. Let $t_{0}$ be any positive number and let $x_{0}$ be any point on $\Gamma\left(t_{0}\right)$. Suppose that $x_{0}$ has positive Lebesgue density with respect to the set $\mathbb{R}^{n} \backslash\left(\Omega\left(t_{0}\right) \cup \bar{G}\right)$. Then there exists an $(x, t)$-neighbourhood $V$ of the point $\left(x_{0}, t_{0}\right)$ such that $\Gamma(t) \cap V$ is an $n$-dimensional $C^{\infty}$ hypersurface transversal to the hyperplane $t=t_{0}$. Moreover, $\theta$ is $C^{\infty}$ in the set $V \cap\{(x, t): x \in \Omega(t) \cup \Gamma(t), t>0\}$.

Corollary 2.5. Radial weak solutions to the Stefan problem (WS) are classical solutions to the Stefan problem (S). 


\section{Hele-Shaw. The Radial Case}

We begin our analysis by considering the simplest case, the behaviour of radial solutions of the Hele-Shaw problem, which can be expressed in explicit form and will be a model for subsequent developments. In this case the free boundary is a sphere which can be expressed in the form $|x|=R(t)$ and the solution is classical.

Radial Hele-Shaw problem. To find functions $R(t)$ and $p(x, t)=p(|x|, t) \geq 0$, defined for $t>0,|x| \geq a$, which satisfy

$$
\begin{cases}\Delta p=0 & \text { in }\{(x, t): a<|x|<R(t), t>0\} \\ p=0 & \text { if }|x|=R(t), t>0 \\ \frac{\partial p}{\partial n}=-L R^{\prime}(t) & \text { if }|x|=R(t), t>0 \\ p=A & \text { if }|x|=a, t>0 \\ R(0)=b . & \end{cases}
$$

3.1. Explicit radial solution. The solution of problem (RHS) can be obtained explicitly as follows.

Space dimension $n \geq 3$. Since $p(\cdot, t)$ is radial and harmonic in $a<|x|<R(t)$, we have

$$
p(x, t)=c_{1}(t)+c_{2}(t)|x|^{2-n} \quad \text { for } a<|x|<R(t) .
$$

We impose the fixed boundary condition, $p(x, t)=A$ if $|x|=a$, and the continuity condition at the free boundary, $p(x, t)=0$ if $|x|=R(t)$, to obtain

$$
p(x, t)=\frac{A\left[|x|^{2-n}-R^{2-n}(t)\right]_{+}}{a^{2-n}-R^{2-n}(t)} .
$$

We finally impose the Stefan condition, which reads

$$
\frac{A(2-n) R^{1-n}(t)}{a^{2-n}-R^{2-n}(t)}=-L R^{\prime}(t)
$$

that is,

$$
A(n-2) / L=a^{2-n} R^{n-1}(t) R^{\prime}(t)-R(t) R^{\prime}(t) .
$$

Integrating this ordinary differential equation over $(0, t)$, we obtain an algebraic equation for $R(t)$ :

$$
\frac{R^{n}(t)}{n a^{n-2}}-\frac{b^{n}}{n a^{n-2}}-\frac{R^{2}(t)}{2}+\frac{b^{2}}{2}=\frac{A(n-2) t}{L} .
$$

This equation has a unique solution $R(t)$ for each $t \geq 0$. Furthermore, it is trivial to see that $R(t)$ is an increasing function such that $\lim _{t \rightarrow \infty} R(t)=\infty$. More precisely, we have

$$
\lim _{t \rightarrow \infty} \frac{R(t)}{R_{\infty}(t)}=1, \quad \text { with } \quad R_{\infty}(t)=c_{\infty} t^{1 / n} \quad \text { and } \quad c_{\infty}=\left(\frac{A a^{n-2} n(n-2)}{L}\right)^{1 / n} .
$$

We note that $p_{t}>0$ for $a<|x|<R(t)$. 
Space dimension $n=2$. Since $p(\cdot, t)$ is radial and harmonic in $a<|x|<R(t)$, we have

$$
p(x, t)=c_{1}(t)+c_{2}(t) \log |x| \quad \text { for } a<|x|<R(t) .
$$

Imposing the fixed boundary condition and the continuity condition at the free boundary, we obtain

$$
p(x, t)=\frac{A[\log R(t)-\log |x|]_{+}}{\log R(t)-\log a} .
$$

We finally impose the Stefan condition, which leads to

$$
A / L=-R R^{\prime} \log a+R R^{\prime} \log R .
$$

Integrating this ordinary differential equation over $(0, t)$, we obtain an algebraic equation for $R(t)$ :

$$
\frac{R^{2}(t)}{2}\left(\log R(t)-\frac{1}{2}-\log a\right)-\frac{b^{2}}{2}\left(\log b-\frac{1}{2}-\log a\right)=\frac{A t}{L} .
$$

This equation has just one solution, $R(t)$, for each $t \geq 0$. It is easy to check that $R(t)$ is an increasing function such that $\lim _{t \rightarrow \infty} R(t)=\infty$. Furthermore,

$$
\lim _{t \rightarrow \infty} \frac{R^{2}(t) \log R(t)}{t}=2 A / L
$$

Taking logarithms, we obtain

$$
\lim _{t \rightarrow \infty} 2 \log R(t)\left(1+\frac{\log \log R(t)}{2 \log R(t)}-\frac{\log t}{2 \log R(t)}\right)=\log \frac{2 A}{L},
$$

and so, necessarily,

$$
\lim _{t \rightarrow \infty} \frac{\log t}{2 \log R(t)}=1
$$

Combining this with (3.6), we get

$$
\lim _{t \rightarrow \infty} \frac{R^{2}(t)}{2(t / \log t)}=\frac{2 A}{L}
$$

and hence

$$
\lim _{t \rightarrow \infty} \frac{R(t)}{R_{\infty}(t)}=1, \quad \text { with } \quad R_{\infty}(t)=c_{\infty}(t / \log t)^{1 / 2} \quad \text { and } \quad c_{\infty}=2 \sqrt{A / L} .
$$

As in the case $n \geq 3$, we have that $p_{t}>0$ if $a<|x|<R(t)$.

3.2. Near field limit. In order to better understand the asymptotic behaviour of these solutions and their use as a model for the Stefan problem, we will perform an analysis of near and far field limits. We understand by near field limit the limit taken for constant $x$ as $t \rightarrow \infty$. We immediately obtain from (3.1) that, in the case $n \geq 3$,

$$
\lim _{t \rightarrow \infty} p(x, t)=P_{A, a}(x) \equiv A a^{n-2}|x|^{2-n},
$$

which is the solution of the Dirichlet exterior problem for the Laplacian:

$$
\begin{cases}\Delta P=0, & |x|>a, \\ P=A, & |x|=a, \\ P(x) \rightarrow 0, & \text { as }|x| \rightarrow \infty .\end{cases}
$$


Indeed, we have the estimate

$$
0 \leq P_{A, a}(x)-p(x, t) \leq \frac{A a^{n-2}}{R^{n-2}(t)-a^{n-2}},
$$

which, in view of the estimate $R(t) \sim c_{\infty} t^{1 / n}$, implies the following convergence result:

Theorem 3.1. Let $p$ be the solution of (RHS) with $n \geq 3$. Then

$$
p(x, t)=P_{A, a}(x)+O\left(t^{(2-n) / n}\right) .
$$

In the case $n=2$ we obtain from (3.4) that

$$
\lim _{t \rightarrow \infty} p(x, t)=A \text {. }
$$

Observe that the constant $A$ is the solution for the Dirichlet exterior problem for the Laplacian:

$$
\begin{cases}\Delta P=0, & |x|>a, \\ P=A, & |x|=a, \\ P(x), & \text { bounded }\end{cases}
$$

Moreover, we have the estimate

$$
0 \leq A-p(x, t) \leq \frac{A \log (|x| / a)}{\log R(t)-\log a},
$$

which, in view of the estimate $R(t) \sim c_{\infty}(t / \log t)^{1 / 2}$, implies the following convergence result:

Theorem 3.2. Let $p$ be the solution of (RHS) with $n=2$. Then, on each compact subset of $\{|x| \geq a\}$,

$$
p(x, t)=A+O(1 / \log t) .
$$

3.3. Far field limit. The far field expansion is given in terms of the new variables

$$
\begin{array}{lll}
v=p t^{(n-2) / n}, & \xi=x t^{-1 / n} & \text { if } n \geq 3, \\
v=p \log R(t), & \xi=x / R(t) & \text { if } n=2,
\end{array}
$$

where $R(t)$ is the solution of (3.5). In view of (3.1) and (3.4) we have the following asymptotic result:

Theorem 3.3. Let $p$ be a solution of problem (RHS) with $n \geq 2$ and let $v$ be its rescaling given by (3.7). Then

$$
\begin{array}{ll}
\lim _{t \rightarrow \infty} v(\xi, t)=A a^{n-2}\left[|\xi|^{2-n}-c_{\infty}^{2-n}\right]_{+} & \text {if } n \geq 3, \\
\lim _{t \rightarrow \infty} v(\xi, t)=A[-\log |\xi|]_{+} & \text {if } n=2,
\end{array}
$$

uniformly on compact subsets of $\mathbb{R}^{n} \backslash\{0\}$, where $c_{\infty}$ has the same meaning as in (3.3).

In terms of the original variables our convergence result can be written as

$$
\begin{array}{ll}
p(x, t) \sim P_{\infty}(x, t)=A a^{n-2}\left[|x|^{2-n}-R_{\infty}^{2-n}(t)\right]_{+} & \text {if } n \geq 3, \\
p(x, t) \sim P_{\infty}(x, t)=\frac{A[\log R(t)-\log |x|]_{+}}{\log R(t)} & \text { if } n=2 .
\end{array}
$$


The functions $P_{\infty}(x, t)$ are solutions of the Hele-Shaw problem in the perforated space $\Omega=\mathbb{R}^{n} \backslash\{0\}$ with a singularity as $|x| \rightarrow 0$ like that of the fundamental solution for the Laplacian.

\section{Near field limit for general Hele-ShaW}

Let $P(x)$ be the solution of the Dirichlet exterior problem for the Laplacian,

$$
\begin{cases}\Delta P=0 & \text { if } x \in \mathbb{R}^{n} \backslash G, \\ P=g & \text { if } x \in \Gamma, \\ \lim _{|x| \rightarrow \infty} P(x)=0 & \text { if } n \geq 3 \\ P(x) \text { bounded } & \text { if } n=2\end{cases}
$$

We have the following convergence result:

Theorem 4.1. Let $n \geq 2$. Then the weak solution $p(x, t)$ of the Hele-Shaw problem converges as $t$ tends to infinity to the unique solution $P(x)$ of the Dirichlet exterior problem (DE) uniformly on compact subsets of $\mathbb{R}^{n} \backslash G$.

We will need some auxiliary results.

Lemma 4.2. Let $G_{1}$ be a domain with smooth boundary satisfying $\bar{G} \subset G_{1} \subset \bar{G}_{1} \subset$ $\bar{G} \cup G_{0}$. Then there exists a constant $\delta>0$ such that $p(x, t) \geq \delta$ for all $x \in \partial G_{1}$, $t \geq 0$.

Proof. Let $\hat{p}$ be the solution to the following boundary value problem:

$$
\begin{cases}\Delta \hat{p}=0, & x \in G_{0}, \\ \hat{p}(x)=g(x), & x \in \Gamma, \\ \hat{p}(x)=0, & x \in \Gamma_{0} .\end{cases}
$$

Then $p(x, t) \geq \hat{p}(x)$ for $x \in G_{0}$. The existence of a constant $\delta>0$ such that $\hat{p}(x) \geq \delta$ for all $x \in \partial G_{1}$ is a consequence of the strong maximum principle.

Lemma 4.3. The free boundary, $\Gamma(t)$, of a weak solution to the Hele-Shaw problem satisfies

$$
\lim _{t \rightarrow \infty} \operatorname{dist}(\Gamma(t), G)=\infty .
$$

Proof. We consider a radial solution $\tilde{p}$ of (RHS) with $a, b$ and $A$ small enough so that $B_{a} \subseteq G, B_{b} \subseteq \bar{G} \cup G_{0}$, and $\tilde{p}(x, t) \leq \delta$ for $x \in \partial G_{1}$, where $\delta$ is the constant provided by Lemma 4.2 and $B_{\rho} \equiv B_{\rho}(0)$. Using comparison in $\mathbb{R}^{n} \backslash G_{1}$, we obtain that $\Omega(t) \cup G \supseteq \operatorname{supp} \tilde{p}(\cdot, t) \cup B_{a}=B_{R(t)}$, with $R(t)$ given by (3.2) or (3.5). Now, $R(t) \rightarrow \infty$ as $t \rightarrow \infty$ and, therefore, the support of $p$ eventually covers every compact subset of $\mathbb{R}^{n} \backslash G$.

Lemma 4.4. There is a constant $C>0$ such that

$$
0 \leq p(x, t) \leq C|x|^{2-n} .
$$

Proof. The function $\hat{p}(x, t)=C|x|^{2-n}$, which is harmonic, is a weak solution to the Hele-Shaw problem (WHS) with $\hat{\chi}=0$, following the notation introduced in Section 2. If we take $C>0$ big enough, then $\hat{p}(x, t) \geq g(x)$ on $\Gamma$. The result follows by comparison (see the remark after Proposition 2.1). 
Proof of Theorem 4.1. The a priori estimate for the pressure given by Lemma 4.4 and the harmonicity of $p$ provide us with uniform (in $t$ ) interior Hölder estimates for $p, D_{i} p$ and $D_{i j} p$. These uniform estimates are valid up to the fixed boundary $\Gamma$, as this hypersurface is smooth. Thus the Ascoli-Arzelà theorem guarantees the existence of a sequence $\left\{t_{n}\right\}$ such that $\left\{p\left(x, t_{n}\right)\right\}$ converges to a certain function $p_{\infty}(x)$ uniformly on compact subsets of $\mathbb{R}^{n} \backslash G$.

The next step is to identify the limit $p_{\infty}$. Using the previous a priori estimates, it is straightforward to see that

$$
\begin{cases}\Delta p_{\infty}(x)=0 & \text { in } \mathbb{R}^{n} \backslash G \\ p_{\infty}(x)=g(x) & \text { if } x \in \Gamma\end{cases}
$$

Moreover, $0 \leq p_{\infty}(x) \leq C|x|^{2-n}$. Thus $\lim _{|x| \rightarrow \infty} p_{\infty}(x)=0$ if $n \geq 3$, and $p_{\infty}$ is bounded if $n=2$. Therefore $p_{\infty}$ is the unique solution of (DE). The uniqueness of the limit implies that convergence is not restricted to a sequence. Hence the result.

In the next lemma we describe the asymptotic behaviour of $P(x)$ as $|x| \rightarrow \infty$.

Lemma 4.5. Let $n \geq 2$. There exists a constant $C_{*}=C_{*}(g, G)$ such that the solution $P$ of problem (DE) satisfies

$$
\lim _{|x| \rightarrow \infty}|x|^{n-2} P(x)=C_{*}(g, G) .
$$

Proof. We consider the inversion mapping $I: x \mapsto x /|x|^{2}$. Let $\Omega^{*}=I\left(\mathbb{R}^{n} \backslash G\right) \cup\{0\}$. Thus $\Omega^{*}$ is the domain bounded by $\partial \Omega^{*}=I(\Gamma)$. Let $P^{*}(x)=|x|^{2-n} P\left(x /|x|^{2}\right)$ be the Kelvin transform of $P$. As $P$ is harmonic in $\mathbb{R}^{n} \backslash G$, so is $P^{*}$ in $\Omega^{*} \backslash\{0\}$. Hence

$$
\begin{cases}\Delta P^{*}=0 & \text { in } \Omega^{*} \backslash\{0\}, \\ P^{*}(x)=|x|^{2-n} g\left(x /|x|^{2}\right) & \text { if } x \in \partial \Omega^{*} .\end{cases}
$$

Moreover, as $0 \leq P(x) \leq C|x|^{2-n}$, then $0 \leq P^{*}(x) \leq C$. Therefore $P^{*}$ cannot be singular at the origin, and hence it is the unique solution of

$$
\begin{cases}\Delta P^{*}=0 & \text { in } \Omega^{*}, \\ P^{*}(x)=|x|^{2-n} g\left(x /|x|^{2}\right) & \text { if } x \in \partial \Omega^{*} .\end{cases}
$$

Now,

$$
\lim _{|x| \rightarrow \infty}|x|^{n-2} P(x)=P^{*}(0) .
$$

This last quantity can be computed explicitly, and is given by

$$
P^{*}(0)=\int_{\partial \Omega^{*}}|x|^{2-n} g\left(\frac{x}{|x|^{2}}\right) \frac{\partial \mathcal{G}^{*}}{\partial \nu}(0, x) d S \equiv C_{*}(g, G),
$$

where $\mathcal{G}^{*}$ is the Green function for the domain $\Omega^{*}$.

\section{FAr field limit for general Hele-ShaW}

We prove that close to the free boundary the solution to the Hele-Shaw problem converges to a radial, self-similar solution in $\mathbb{R}^{n} \backslash\{0\}$ of the same problem, compactly supported in $x$ and with a singularity as $|x| \rightarrow 0$ like that of the fundamental solution for the Laplacian. We would like to remark that the far field limit inherits asymptotic information from the near field limit. Namely, in the far field limit 
there is a free constant which is determined through a suitable matching of both developments.

Theorem 5.1. Let $p$ be the weak solution to the Hele-Shaw problem (WHS) and let $C_{*}=C_{*}(g, G)$ be the constant given by Lemma 4.5 .

A) If $n \geq 3$ then

$$
\lim _{t \rightarrow \infty} t^{(n-2) / n}|p(x, t)-\mathcal{P}(x, t)|=0
$$

uniformly on sets of the form $\left\{x \in \mathbb{R}^{n}:|x| \geq \delta t^{1 / n}\right\}, \delta>0$, where

$$
\mathcal{P}(x, t)=C_{*}\left[|x|^{2-n}-\left(C_{*} n(n-2) t / L\right)^{(2-n) / n}\right]_{+} .
$$

B) If $n=2$ then

$$
\lim _{t \rightarrow \infty} \log \mathcal{R}(t)\left|p(x, t)-\mathcal{P}_{2}(x, t)\right|=0
$$

uniformly on sets of the form $\left\{x \in \mathbb{R}^{n}:|x| \geq \delta \mathcal{R}(t)\right\}, \delta>0$, where $\mathcal{R}(t)$ is the unique solution for $t>0$ of

$$
\frac{\mathcal{R}^{2}}{2}\left(\log \mathcal{R}-\frac{1}{2}\right)=\frac{C_{*} t}{L}
$$

and

$$
\mathcal{P}_{2}(x, t)=\frac{C_{*}[\log \mathcal{R}(t)-\log |x|]_{+}}{\log \mathcal{R}(t)} .
$$

Remark. As $t \rightarrow \infty, \mathcal{R}(t) \sim 2 \sqrt{C_{*} / L}(t / \log t)^{1 / 2}$.

Proof. Using Lemma 4.5 we deduce that, given $\varepsilon>0$, there exists a value $a \gg 1$ such that

$$
\left|P(x)-\frac{C_{*}}{|x|^{n-2}}\right|<\frac{\varepsilon}{2|x|^{n-2}} \leq \frac{\varepsilon}{2 a^{n-2}} \leq \frac{\varepsilon}{2}
$$

if $|x| \geq a$. Now, Theorem 4.1 guarantees that there exists a value $T>0$ such that, for all $t \geq T$ and $|x|=a$,

$$
|p(x, t)-P(x)|<\frac{\varepsilon}{2} .
$$

Combining these two facts, we get that, for all $t \geq T$ and $|x|=a$,

$$
\left|p(x, t)-\frac{C_{*}}{|x|^{n-2}}\right|<\varepsilon .
$$

Let $m_{+}(t)=\max _{x \in \Gamma(t)}|x|, m_{-}(t)=\min _{x \in \Gamma(t)}|x|$. It is clear (taking, if necessary, a larger value of $T$ ) that $m_{+}(t) \geq m_{-}(t)>a$ for every $t>T$. Let $p^{ \pm}$be the solutions to the radial Hele-Shaw problem (RHS) for $t \geq T$, with boundary data $p^{ \pm}=\left(C_{*} / a^{n-2}\right) \pm \varepsilon$ at $|x|=a$, and support radii $m_{ \pm}(T)$ at time $t=T$. Let $R_{ \pm}(t)$ be the radii of their supports at time $t$. Using comparison for $|x| \geq a, t \geq T$, we get that

$$
p^{-}(x, t) \leq p(x, t) \leq p^{+}(x, t),
$$

for $|x| \geq a, t \geq T$.

A) For $n \geq 3$ we consider the rescaling transformation

$$
p_{\lambda}(x, t)=\lambda^{(n-2) / n} p\left(\lambda^{1 / n} x, \lambda t\right) .
$$


A simple calculation shows that

$$
\begin{gathered}
\left|p_{\lambda}(\xi, 1)-\mathcal{P}(\xi, 1)\right|=\lambda^{(n-2) / n}\left|p\left(\lambda^{1 / n} \xi, \lambda\right)-\mathcal{P}\left(\lambda^{1 / n} \xi, \lambda\right)\right| \\
=\lambda^{(n-2) / n}|p(x, \lambda)-\mathcal{P}(x, \lambda)|,
\end{gathered}
$$

where we have used the invariance of $\mathcal{P}$ with respect to the rescaling and the notation $x=\lambda^{1 / n} \xi$. It clearly follows that the uniform convergence of $p_{\lambda}(\xi, 1)$ to $\mathcal{P}(\xi, 1)$ as $\lambda \rightarrow \infty$ on sets of the form $\{|\xi| \geq \delta\}$ is equivalent to the stated convergence (5.1).

Taking now $x=\xi \lambda^{1 / n}$ and $t=\lambda$ in (5.3) and multiplying the resulting inequalities by $\lambda^{(n-2) / n}$, we obtain

$$
p_{\lambda}^{-}(\xi, 1) \leq p_{\lambda}(\xi, 1) \leq p_{\lambda}^{+}(\xi, 1)
$$

for $|\xi| \geq a / \lambda^{1 / n}, \lambda \geq T$. But,

$$
\begin{gathered}
\lim _{\lambda \rightarrow \infty} p_{\lambda}^{ \pm}(\xi, 1)=\lim _{\lambda \rightarrow \infty} \frac{\left(C_{*} a^{2-n} \pm \varepsilon\right)\left[|\xi|^{2-n}-\left(R_{ \pm}(\lambda) / \lambda^{1 / n}\right)^{2-n}\right]_{+}}{a^{2-n}-R_{ \pm}^{2-n}(\lambda)} \\
=\left(C_{*} \pm \varepsilon a^{n-2}\right)\left[|\xi|^{2-n}-c_{ \pm}^{2-n}\right]_{+},
\end{gathered}
$$

uniformly on sets of the form $\{\xi:|\xi| \geq \delta\}, \delta>0$, where

$$
c_{ \pm}=\left(\left(C_{*} \pm \varepsilon a^{n-2}\right) n(n-2) / L\right)^{1 / n} .
$$

Therefore, given $\tilde{\varepsilon}>0$ and $\delta>0$, there is a value $\Lambda$ such that for $|\xi| \geq \delta$ and $\lambda \geq \Lambda$ we have

$$
\left|p_{\lambda}(\xi, 1)-\mathcal{P}(\xi, 1)\right|<\tilde{\varepsilon},
$$

which is the required uniform convergence result.

B) When $n=2$ we consider the rescaling transformation

$$
p_{\lambda}(x, t)=\log \mathcal{R}(\lambda) p(\mathcal{R}(\lambda) x, \lambda t) .
$$

A simple calculation shows that

$$
\left|p_{\lambda}(\xi, 1)-C_{*}[-\log |\xi|]_{+}\right|=\log \mathcal{R}(\lambda)\left|p(x, \lambda)-\mathcal{P}_{2}(x, \lambda)\right|,
$$

where we have used the notation $x=\mathcal{R}(\lambda) \xi$. It follows immediately that the uniform convergence of $p_{\lambda}(\xi, 1)$ to $C_{*}[-\log |\xi|]_{+}$as $\lambda \rightarrow \infty$ on sets of the form $\{|\xi| \geq \delta\}$ is equivalent to the stated convergence (5.2).

Now, taking $x=\mathcal{R}(\lambda) \xi$ and $t=\lambda$ in (5.3) and multiplying the resulting inequalities by $\log \mathcal{R}(\lambda)$, we obtain

$$
p_{\lambda}^{-}(\xi, 1) \leq p_{\lambda}(\xi, 1) \leq p_{\lambda}^{+}(\xi, 1)
$$

for $|\xi| \geq a / \mathcal{R}(\lambda), \lambda \geq T$. But,

$$
\begin{gathered}
\lim _{\lambda \rightarrow \infty} p_{\lambda}^{ \pm}(\xi, 1)=\lim _{\lambda \rightarrow \infty} \frac{\left(C_{*} \pm \varepsilon\right)\left[\log \left(R_{ \pm}(\lambda) / \mathcal{R}(\lambda)\right)-\log |\xi|\right]_{+}}{\frac{\log R_{ \pm}(\lambda)}{\log \mathcal{R}(\lambda)}-\frac{\log a}{\log \mathcal{R}(\lambda)}} \\
=\left(C_{*} \pm \varepsilon\right)\left[\frac{1}{2} \log \frac{C_{*} \pm \varepsilon}{C_{*}}-\log |\xi|\right]_{+}, \\
\lim _{\varepsilon \rightarrow 0}\left(C_{*} \pm \varepsilon\right)\left[\frac{1}{2} \log \frac{C_{*} \pm \varepsilon}{C_{*}}-\log |\xi|\right]_{+}=C_{*}[-\log |\xi|]_{+},
\end{gathered}
$$


uniformly on sets of the form $\{\xi:|\xi| \geq \delta\}, \delta>0$. Therefore we get that, given $\tilde{\varepsilon}>0$ and $\delta>0$, there is a value $\Lambda$ such that for $|\xi| \geq \delta$ and $\lambda \geq \Lambda$ we have

$$
\left|p_{\lambda}(\xi, 1)-C_{*}[-\log |\xi|]_{+}\right|<\tilde{\varepsilon},
$$

which is the required uniform convergence result.

We also prove that the free boundary approaches a sphere with a precise radius growth.

Theorem 5.2. Let $m_{+}(t)=\max _{x \in \Gamma(t)}|x|$ and $m_{-}(t)=\min _{x \in \Gamma(t)}|x|$, where $\Gamma(t)$ is the free boundary of the solution of the Hele-Shaw problem. Then

$$
\begin{array}{ll}
\lim _{t \rightarrow \infty} \frac{m_{ \pm}(t)}{t^{1 / n}}=\left(C_{*} n(n-2) / L\right)^{1 / n} & \text { if } n \geq 3, \\
\lim _{t \rightarrow \infty} \frac{m_{ \pm}(t)}{(t / \log t)^{1 / 2}}=2\left(C_{*} / L\right)^{1 / 2} & \text { if } n=2 .
\end{array}
$$

Proof. We prove the case $n \geq 3$. The case $n=2$ is completely analogous. From (5.3) we get that

$$
\frac{R_{-}(t)}{t^{1 / n}} \leq \frac{m_{-}(t)}{t^{1 / n}} \leq \frac{m_{+}(t)}{t^{1 / n}} \leq \frac{R_{+}(t)}{t^{1 / n}}
$$

On the other hand,

$$
\lim _{t \rightarrow \infty} \frac{R_{ \pm}(t)}{t^{1 / n}}=\left(\left(C_{*} \pm \varepsilon a^{n-2}\right) n(n-2) / L\right)^{1 / n} .
$$

As $\varepsilon$ is arbitrary, the result follows.

\section{FAR FIELD LIMIT FOR RADIAL SOLUTIONS TO THE STEFAN PROBlem FOR $n \geq 3$}

In the remaining sections we prove that, if the space dimension is greater than one, solutions of the Stefan problem in an external domain behave asymptotically exactly as solutions of the Hele-Shaw problem with the same boundary data. As $t \rightarrow \infty$ any trace of the initial data is lost. We apply the same approach that was developed in Sections 4 and 5 for the Hele-Shaw problem. There is a small difference, as in this case we do not know explicit radial solutions. However, we manage to prove that, if $n \geq 2$, radial solutions of the Stefan problem converge near the free boundary to compactly supported in $x$, radial and self-similar solutions in $\mathbb{R}^{n} \backslash\{0\}$ to Hele-Shaw, with a singularity as $|x| \rightarrow 0$ like that of the fundamental solution for the Laplacian. This will be enough to study the far field limit of general solutions.

We begin with the study of the far field limit for radial solutions. In this section, after giving some preliminary results about radial solutions, we consider the far field limit for this kind of solutions in the case $n \geq 3$. The case $n=2$ is slightly different and will be dealt with in Section 7. General solutions are studied in Section 8, where we analyze both the near and far field limits.

Let us first derive some general facts for radial solutions. The free boundary is obviously a sphere, which can be expressed in the form $|x|=R(t)$, and the solution is classical. The regularity of the free boundary makes the analysis much easier. 
The problem consists of finding functions $R(t) \geq b$ and $\theta(x, t)=\theta(|x|, t) \geq 0$, defined for $t>0,|x| \geq a$, which satisfy

$$
\begin{cases}\frac{\partial \theta}{\partial t}=\Delta \theta & \text { if } a<|x|<R(t), t>0 \\ \theta=0 & \text { if }|x|=R(t), t>0 \\ \frac{\partial \theta}{\partial n}=-L R^{\prime}(t) & \text { if }|x|=R(t), t>0 \\ \theta=A & \text { if }|x|=a, t>0 \\ \theta(x, 0)=\theta_{0}(|x|) & \text { if }|x| \geq a \\ R(0)=b . & \end{cases}
$$

Radial solutions to the Stefan problem can be compared with radial solutions to the Hele-Shaw problem.

Proposition 6.1. Let $p$ and $\theta$ be radial solutions to the Hele-Shaw problem and to the Stefan problem respectively, and let $|x|=R_{p}(t),|x|=R_{\theta}(t)$ be the corresponding interfaces. If $R_{p}(0)>R_{\theta}(0), p(x, 0) \geq \theta(x, 0)$ and, moreover, $p(x, t) \geq \theta(x, t)$ on the fixed boundary, that is, for $|x|=a, t>0$, then $p(x, t) \geq \theta(x, t)$ for all $|x| \geq a$ and $t \geq 0$.

Proof. Let $T$, which may be infinite, be the first time when $R_{p}=R_{\theta}$. Because of the continuity of the free boundary of both problems, $T>0$. As we pointed out in Section 3, $p_{t}>0$ for $a<|x|<R_{p}(t)$. Therefore $p$ is a supersolution of the heat equation in that set. Applying comparison in $a \leq|x| \leq R_{\theta}(t), 0 \leq t \leq T$, we obtain that $p(x, t) \geq \theta(x, t)$ for $0 \leq t \leq T$.

We now prove that $T=\infty$. Suppose it were finite. Hopf's lemma would imply that

$$
\frac{\partial p}{\partial n}<\frac{\partial \theta}{\partial n} \quad \text { for }|x|=R_{p}(T)=R_{\theta}(T), t=T .
$$

Using the dynamical condition at the free boundary, we would obtain $R_{p}^{\prime}(T)>$ $R_{\theta}^{\prime}(T)$, a contradiction.

This immediately leads to an estimate from above for the free boundary of radial solutions to the Stefan problem.

Corollary 6.2. Let $|x|=R(t)$ be the free boundary of a radial solution to the Stefan problem. There are constants $C, T>0$, such that, for all $t \geq T$,

$$
\begin{array}{llrl}
R(t) & \leq C t^{1 / n}, & & \geq 3, \\
R(t) & \leq C(t / \log t)^{1 / 2}, & & n=2 .
\end{array}
$$

We shall also need an estimate from above for the temperature. This estimate is not restricted to radial solutions.

Lemma 6.3. Let $\theta$ be a weak solution to the Stefan problem for $n \geq 2$. There is a constant $C>0$ such that

$$
0 \leq \theta(x, t) \leq C|x|^{2-n} .
$$

Proof. The function $\hat{\theta}(x, t)=C|x|^{2-n}$, which is a stationary solution of the heat equation, is also a weak solution to the Stefan problem (WS) with $\hat{f}=C|x|^{2-n}$, in 
the notation of Section 2. If we take $C>0$ big enough, then $\hat{f}(x) \geq h(x) \geq f(x)$, and $\hat{\theta}(x, t) \geq g(x)$ on $\Gamma$. Applying Proposition 2.2 now gives us the result.

In order to obtain a more precise description of the asymptotic behaviour of the free boundary, we need to transfer some information from the near region to the far zone. The basic transfer tool is the identity

$$
\begin{aligned}
\int_{\rho \leq|x| \leq R(t)} & \left(\theta(x, t)-\theta_{0}(|x|)\right) d x \\
= & -L \omega_{n}\left(R^{n}(t)-R^{n}(s(\rho))\right)-\left.n \omega_{n} \rho^{n-1} \int_{s(\rho)}^{t} \frac{\partial \theta}{\partial n}(x, \tau)\right|_{|x|=\rho} d \tau,
\end{aligned}
$$

where $\omega_{n}$ is the measure of the unit ball in $\mathbb{R}^{n}, a \leq \rho \leq R(t)$ and

$$
s(\rho)= \begin{cases}R^{-1}(\rho) & \text { if } \rho \geq b, \\ 0 & \text { if } a \leq \rho \leq b,\end{cases}
$$

so that $R(s(\rho))=\max (\rho, b)$. To obtain identity (6.1) we integrate the equation over the set $\{(x, \tau): \rho \leq|x| \leq R(\tau), s(\rho)<\tau<t\}$ and apply Gauss' theorem and the dynamical condition at the free boundary.

For the rest of the section we restrict ourselves to the case $n \geq 3$.

Theorem 6.4. Let $|x|=R(t)$ be the free boundary of the radial solution to the Stefan problem (RS) with $n \geq 3$. Then

$$
\lim _{t \rightarrow \infty} \frac{R(t)}{t^{1 / n}}=\left(A a^{n-2} n(n-2) / L\right)^{1 / n} .
$$

Proof. Dividing (6.1) by $t \rho^{n-1}$ and integrating over $a \leq \rho \leq R(t)$, we obtain

$$
\begin{aligned}
& \frac{1}{t} \int_{a}^{R(t)} \frac{1}{\rho^{n-1}}\left(\int_{\rho \leq|x| \leq R(t)}\left(\theta(x, t)-\theta_{0}(|x|)\right) d x\right) d \rho \\
= & \frac{L \omega_{n} R^{n}(t)}{(n-2) t}\left(R^{2-n}(t)-a^{2-n}\right)+\frac{L \omega_{n} b^{n}}{(n-2) t}\left(a^{2-n}-b^{2-n}\right) \\
+ & \frac{L \omega_{n}}{t}\left(\frac{R^{2}(t)}{2}-\frac{b^{2}}{2}\right)+n \omega_{n} A .
\end{aligned}
$$

If we let $t \rightarrow \infty$, the left-hand side tends to zero while the right-hand side tends to

$$
-\frac{L \omega_{n} a^{2-n}}{(n-2)} \lim _{t \rightarrow \infty} \frac{R^{n}(t)}{t}+n \omega_{n} A .
$$

To see this we just use the estimate from above for the free boundary given in Corollary 6.2 and the bound for $\theta$ given in Lemma 6.3.

Remark. Formula (6.2) shows that the free boundary inherits precise asymptotic information from the fixed boundary.

We next use this information about the asymptotic behaviour of the free boundary to obtain the large-time behaviour of the temperature in the far field region.

Theorem 6.5. Let $\theta$ be the radial solution to the Stefan problem (RS) with $n \geq 3$. Then

$$
\lim _{t \rightarrow \infty} t^{(n-2) / n}|\theta(x, t)-\mathcal{P}(x, t)|=0
$$


uniformly on sets of the form $\left\{x \in \mathbb{R}^{n}:|x| \geq \delta t^{1 / n}\right\}, \delta>0$, where

$$
\mathcal{P}(x, t)=A a^{n-2}\left[|x|^{2-n}-R_{\infty}^{2-n} t^{(2-n) / n}\right]_{+}, \quad R_{\infty}^{n}=A a^{n-2} n(n-2) / L .
$$

Remark. Compare this formula with (3.1), (3.3).

Proof. We use the technique of continuous rescaling. We define the rescaled temperature

$$
v(\xi, \tau)=t^{(n-2) / n} \theta\left(\xi t^{1 / n}, t\right), \quad \tau=\frac{n t^{(n-2) / n}}{n-2} .
$$

This function $v$ satisfies the equation

$$
v_{\tau}=\Delta v+\frac{1}{\tau}\left(v+\frac{1}{(n-2)} \nabla v \cdot \xi\right)
$$

in the domain $\alpha(\tau) \leq|\xi| \leq R_{v}(\tau), \tau>0$, where $\alpha(\tau)=a / t^{1 / n}$ and $R_{v}(\tau)=$ $R(t) / t^{1 / n}$. Moreover,

$$
\begin{array}{ll}
v(\xi, \tau)=0 & \text { if }|\xi|=R_{v}(\tau), \\
v(\xi, \tau)=A t^{(n-2) / n} & \text { if }|\xi|=\alpha(\tau) .
\end{array}
$$

Theorem 6.4 implies that $\lim _{\tau \rightarrow \infty} R_{v}(\tau)=R_{\infty}$. Hence, given $\tilde{\varepsilon}>0$, there is a value $T$ such that for $\tau \geq T$

$$
R_{\infty}-\tilde{\varepsilon} \leq R_{v}(\tau) \leq R_{\infty}+\tilde{\varepsilon} .
$$

We shall assume without loss of generality that $T>1$.

We consider the solutions, $v^{ \pm}$, of $(6.4)$ in $S_{ \pm} \equiv\left\{(\xi, \tau): \alpha(\tau)<|\xi|<R_{\infty} \pm \tilde{\varepsilon}, \tau\right\rangle$ $T\}$ with boundary data

$$
\begin{array}{ll}
v^{ \pm}(\xi, \tau)=0 & \text { if }|\xi|=R_{\infty} \pm \tilde{\varepsilon}, \tau \geq T, \\
v^{ \pm}(\xi, \tau)=A t^{(n-2) / n} & \text { if }|\xi|=\alpha(\tau), \tau \geq T,
\end{array}
$$

and continuous initial data at time $\tau=T$ such that

$$
\begin{array}{ll}
v(\xi, T) \leq v^{+}(\xi, T) & \text { if } \alpha(T) \leq|\xi| \leq R_{\infty}+\tilde{\varepsilon}, \\
v(\xi, T) \geq v^{-}(\xi, T) \geq 0 & \text { if } \alpha(T) \leq|\xi| \leq R_{\infty}-\tilde{\varepsilon} .
\end{array}
$$

Comparison shows that $v \geq v^{-}$in $S^{-}$and that $v \leq v^{+}$in $S^{+}$.

Next we prove a lemma that gives the asymptotic behaviour of $v^{ \pm}$.

Lemma 6.6. Let $v$ be any solution of (6.4) in $S \equiv\{(\xi, \tau): \alpha(\tau)<|\xi|<d, \tau>T\}$ with boundary data

$$
\begin{array}{ll}
v(\xi, \tau)=0 & \text { if }|\xi|=d, \tau \geq T, \\
v(\xi, \tau)=A t^{(n-2) / n} & \text { if }|\xi|=\alpha(\tau), \tau \geq T,
\end{array}
$$

and continuous initial data at time $\tau=T$. Then $v$ converges to

$$
w=A a^{n-2}\left(|\xi|^{2-n}-d^{2-n}\right)_{+}
$$

uniformly on compact subsets of $B_{d} \backslash\{0\}$. 
Proof. (i) An easy computation leads to

$$
w_{\tau}-\Delta w-\frac{1}{\tau}\left(w+\frac{1}{(n-2)} \nabla w \cdot \xi\right)=\frac{A a^{n-2} d^{2-n}}{\tau} .
$$

Thus, if we define $\phi=w-v$, we have

$$
\phi_{\tau}-\Delta \phi-\frac{1}{\tau}\left(\phi+\frac{1}{(n-2)} \nabla \phi \cdot \xi\right)=\frac{A a^{n-2} d^{2-n}}{\tau}
$$

in $S$, and moreover

$$
\begin{array}{ll}
\phi(\xi, \tau)=0 & \text { if }|\xi|=d, \tau \geq T, \\
\phi(\xi, \tau)=-A a^{(n-2) / n} d^{2-n} & \text { if }|\xi|=\alpha(\tau), \tau \geq T .
\end{array}
$$

Thus we have that $\phi$ is bounded on the parabolic boundary of $S$. We want to prove that in fact $\phi$ is bounded in $S$. Due to the sign of the zero-order term we cannot apply the maximum principle directly, and we need to make an additional argument.

We first control $\psi=\phi / \tau$. This function satisfies

$$
L(\psi) \equiv \psi_{\tau}-\Delta \psi-\frac{1}{(n-2) \tau} \nabla \psi \cdot \xi=\frac{A a^{n-2} d^{2-n}}{\tau^{2}} .
$$

Moreover, $\psi$ is bounded on the parabolic boundary of $S$.

Let $\hat{\psi}=-C$, with $C>0$. Then $L(\hat{\psi})=0<L(\psi)$, and also $\hat{\psi} \leq \psi$ on the parabolic boundary if we take $C$ big enough. Thus, $\psi \geq-C$ in $S$. If we now set $\tilde{\psi}=-C_{1}|\xi|^{2}+C_{2}$, with $C_{1}, C_{2}>0$, we have

$$
L(\tilde{\psi})=2 n C_{1}+\frac{2 C_{1}|\xi|^{2}}{(n-2) \tau}>L(\psi)
$$

just taking $C_{1}$ big enough. Then we choose $C_{2}$ big enough so that $\tilde{\psi} \geq \psi$ on the parabolic boundary. Therefore $\psi \leq \tilde{\psi} \leq C_{2}$ in $S$, and hence $\psi$ is bounded.

If we go back to the equation satisfied by $\phi$, we see that

$$
L(\phi)=\psi+\frac{A a^{n-2} d^{2-n}}{\tau} \equiv f(\xi, \tau) .
$$

Thanks to the estimate for $\psi$ that we have just obtained we have that $|f| \leq C$ in $S$.

Set $\hat{\phi}=C_{1}|\xi|^{2}-C_{2}$, with $C_{1}, C_{2}>0$. Then we obtain

$$
L(\hat{\phi})=-2 n C_{1}-\frac{2 C_{1}|\xi|^{2}}{(n-2) \tau} \leq-C \leq L(\phi)
$$

just taking $C_{1}$ big enough, independent of $C_{2}$. On the other hand, we can take $C_{2}$ big enough so that $\hat{\phi} \leq \phi$ on the parabolic boundary. Therefore $\phi \geq \hat{\phi} \geq-C_{2}$ in $S$. If we now set $\tilde{\phi}=-C_{1}|\xi|^{2}+C_{2}$, with $C_{1}, C_{2}>0$, we have

$$
L(\tilde{\phi})=2 n C_{1}+\frac{2 C_{1}|\xi|^{2}}{(n-2) \tau}>C \geq L(\phi),
$$

just choosing $C_{1}$ big enough. Then we take $C_{2}$ big enough so that $\tilde{\phi} \geq C$ on the parabolic boundary. Therefore $\phi \leq \tilde{\phi} \leq C_{2}$ in $S$. Summarizing: there is a constant $M>0$ such that

$$
|\phi| \leq M
$$


(ii) Let us now prove that $\phi$ tends to zero. Let $r_{0}$ be any number such that $0<r_{0}<d$. We take $\widetilde{T} \geq T$ such that $\alpha(\tau)<r_{0}$ for $\tau \geq \widetilde{T}$. We consider the solutions $\hat{\phi}_{ \pm}$to $(6.5)$ with boundary data

$$
\begin{array}{ll}
\hat{\phi}_{ \pm}(\xi, \tau)=0 & \text { if }|\xi|=d, \tau \geq \widetilde{T} \\
\hat{\phi}_{ \pm}(\xi, \tau)= \pm M & \text { if }|\xi|=r_{0}, \tau \geq \widetilde{T}
\end{array}
$$

and continuous initial data at time $\tau=\widetilde{T}$ such that

$$
\hat{\phi}_{+}(\xi, \widetilde{T}) \geq \phi(\xi, \widetilde{T}), \quad \hat{\phi}_{-}(\xi, \widetilde{T}) \leq \phi(\xi, \widetilde{T}) .
$$

The maximum principle implies that

$$
\hat{\phi}_{-}(\xi, \tau) \leq \phi(\xi, \tau) \leq \hat{\phi}_{+}(\xi, \tau) \text { for } r_{0} \leq|\xi| \leq d, \tau \geq \widetilde{T} .
$$

On the other hand, it is easy to prove (see for example Theorem 2, Chapter 6 in F]) that

$$
\hat{\phi}_{ \pm} \longrightarrow \pm M\left(\frac{|\xi|^{2-n}-d^{2-n}}{r_{0}^{2-n}-d^{2-n}}\right)
$$

as $\tau \rightarrow \infty$ uniformly in $r_{0} \leq|\xi| \leq d$. Thus we have that, given $\varepsilon>0$, there is a number $T_{1} \geq \widetilde{T}$ such that

$$
|\phi(\xi, \tau)| \leq M\left(\frac{|\xi|^{2-n}-d^{2-n}}{r_{0}^{2-n}-d^{2-n}}\right)+\varepsilon
$$

for $\tau \geq T_{1}$ and $r_{0} \leq|\xi| \leq d$.

Suppose that we are given a certain value $0<\delta \leq d$ and a certain $\varepsilon>0$. We take $r_{0}, 0<r_{0}<\delta$, small enough so that

$$
M\left(\frac{\delta^{2-n}-d^{2-n}}{r_{0}^{2-n}-d^{2-n}}\right)<\varepsilon .
$$

Then, taking into account (6.6), we have that there is a value $T_{1} \gg 1$ such that

$$
|\phi(\xi, \tau)| \leq M\left(\frac{\delta^{2-n}-d^{2-n}}{r_{0}^{2-n}-d^{2-n}}\right)+\varepsilon
$$

for $\tau \geq T_{1}$ and $\delta \leq|\xi| \leq d$, i.e.,

$$
|\phi(\xi, \tau)| \leq 2 \varepsilon
$$

Continuation of the proof of Theorem 6.5. Applying Lemma 6.6 to $v^{ \pm}$, we find that, given $\varepsilon, \delta>0$, for each $\tilde{\varepsilon}$ there is a value $T$ such that

$$
\left|v^{ \pm}-A a^{n-2}\left[|\xi|^{n-2}-\left(R_{\infty} \pm \tilde{\varepsilon}\right)^{2-n}\right]_{+}\right|<\varepsilon \quad \text { if } \delta \leq|\xi| \leq 1 \pm \tilde{\varepsilon}
$$

for all $\tau \geq T$. Thus, taking $\tilde{\varepsilon}$ small enough, we have

$$
\begin{aligned}
v(\xi, \tau) & \leq v^{+}(\xi, \tau) \leq A a^{n-2}\left[|\xi|^{2-n}-\left(R_{\infty}+\tilde{\varepsilon}\right)^{2-n}\right]_{+}+\varepsilon \\
& \leq A a^{n-2}\left[|\xi|^{2-n}-R_{\infty}^{2-n}\right]_{+}+2 \varepsilon \\
v(\xi, \tau) & \geq v^{-}(\xi, \tau) \geq A a^{n-2}\left[|\xi|^{2-n}-\left(R_{\infty}-\tilde{\varepsilon}\right)^{2-n}\right]_{+}-\varepsilon \\
& \geq A a^{n-2}\left[|\xi|^{2-n}-R_{\infty}^{2-n}\right]_{+}-2 \varepsilon
\end{aligned}
$$


for all $|\xi| \geq \delta$ and $\tau \geq T$. Note that in order for the last inequality to be true for $|\xi| \geq R_{\infty}-\tilde{\varepsilon}$ we only need to take $\tilde{\varepsilon}$ small enough so that $A a^{n-2}\left(|\xi|^{2-n}-R_{\infty}^{2-n}\right)_{+}-$ $2 \varepsilon \leq 0$ for $|\xi| \geq R_{\infty}-\tilde{\varepsilon}$.

Summarizing: given $\varepsilon>0$ and $\delta>0$, there exists a value $T$ such that for all $|\xi|>\delta$ and $\tau \geq T$,

$$
\left|v-A a^{n-2}\left[|\xi|^{2-n}-R_{\infty}^{2-n}\right]_{+}\right| \leq \varepsilon .
$$

Translating this to the original variables $x$ and $t$, we obtain the stated uniform convergence (6.3).

\section{FAR FIELD LIMIT FOR RADIAL SOLUTIONS TO THE STEFAN PROBLEM FOR $n=2$}

In this section we obtain the asymptotic behaviour of both the free boundary and the temperature in the region close to the free boundary in the two-dimensional case.

Theorem 7.1. Let $|x|=R(t)$ be the free boundary of the radial solution to the Stefan problem (RS) with $n=2$. Then

$$
\lim _{t \rightarrow \infty} \frac{R(t)}{(t / \log t)^{1 / 2}}=2 \sqrt{A / L} .
$$

Proof. Dividing (6.1) by $t \rho$ and integrating over $a \leq \rho \leq R(t)$, we obtain

$$
\begin{gathered}
\frac{1}{t} \int_{a}^{R(t)} \frac{1}{\rho}\left(\int_{\rho \leq|x| \leq R(t)}\left(\theta(x, t)-\theta_{0}(|x|)\right) d x\right) d \rho \\
=-\frac{L \pi R^{2}(t)}{t}(\log R(t)-\log a)+\frac{L \pi}{t}\left(b^{2} \log \frac{b}{a}+\frac{R^{2}(t)}{2}-\frac{b^{2}}{2}\right)+2 \pi A .
\end{gathered}
$$

If we let $t \rightarrow \infty$, the left-hand side tends to zero (we will check this in detail), while the right-hand side tends to

$$
-L \pi \lim _{t \rightarrow \infty} \frac{R^{2}(t) \log R(t)}{t}+2 \pi A .
$$

Thus,

$$
\lim _{t \rightarrow \infty} \frac{R^{2}(t) \log R(t)}{t}=2 A / L
$$

from which it is immediate to obtain (7.1).

To see that the left-hand side of (7.2) tends to zero it is not enough to use the fact that the temperature is bounded plus the estimate from above for the free boundary given by Corollary 6.2. Indeed, because $\theta$ is bounded, the absolute value of the left hand side of (7.2) is estimated above by

$$
\frac{C}{t} \int_{a}^{R(t)} \frac{1}{\rho}\left(R^{2}(t)-\rho^{2}\right) d \rho=\frac{C}{t}\left(R^{2}(t) \log \frac{R(t)}{a}-\frac{R^{2}(t)}{2}+\frac{a^{2}}{2}\right) .
$$

By Proposition 6.1 we know that there exists a radial solution to Hele-Shaw of the form

$$
p(x, t)=\frac{C_{0}[\log (\bar{R}(t) /|x|)]_{+}}{\log (\bar{R}(t) / a)}
$$


such that $p(x, t) \geq \theta(x, t)$. Using the estimates for the radial Hele-Shaw problem, we conclude that the second member of (7.3) is bounded. In order to prove that the left-hand side of (7.2) goes to zero as $t \rightarrow \infty$ we use the bound $\theta(x, t) \leq p(x, t)$ to get

$$
\begin{aligned}
\mid \frac{1}{t} \int_{a}^{R(t)} & \frac{1}{\rho}\left(\int_{\rho \leq|x| \leq R(t)}\left(\theta(x, t)-\theta_{0}(|x|)\right) d x\right) d \rho \mid \\
\leq & \frac{C_{1}}{t \log (\bar{R}(t) / a)} \int_{a}^{R(t)} \frac{1}{\rho}\left(\int_{\rho \leq|x| \leq R(t)} \log (\bar{R}(t) /|x|) d x\right) d \rho \\
\leq & \frac{C_{2}}{t \log (\bar{R}(t) / a)} \int_{a}^{R(t)} \frac{R^{2}(t) \log (\bar{R}(t) / R(t))}{\rho} d \rho \\
& \quad+\frac{C_{2}}{t \log (\bar{R}(t) / a)} \int_{a}^{R(t)}\left(\rho\left(\log (\rho / \bar{R}(t))-\frac{1}{2}\right)+\frac{R^{2}(t)}{2 \rho}\right) d \rho .
\end{aligned}
$$

It is easily seen that the second term of the last member tends to zero. It only remains to be proved that the first term,

$$
\frac{C_{2}}{t \log (\bar{R}(t) / a)} \int_{a}^{R(t)} R^{2}(t) \log \left(\frac{\bar{R}(t)}{R(t)}\right) \frac{d \rho}{\rho}=\frac{C_{3} R^{2}(t) \log (\bar{R}(t) / R(t)) \log (R(t) / a)}{t \log (\bar{R}(t) / a)},
$$

tends to zero. If we prove that $R(t) / \bar{R}(t) \geq C_{4}>0$, the result follows. If this inequality were not true, then there would be a subsequence $\left\{t_{n}\right\}$ such that $R\left(t_{n}\right) / \bar{R}\left(t_{n}\right) \rightarrow 0$. Taking $t=t_{n}$ in (7.3) and letting $n \rightarrow \infty$, this would tend to

$$
C \lim _{n \rightarrow \infty} \frac{R^{2}\left(t_{n}\right) \log R\left(t_{n}\right)}{t_{n}}=C^{\prime} \lim _{n \rightarrow \infty} \frac{R^{2}\left(t_{n}\right) \log R\left(t_{n}\right)}{\bar{R}^{2}\left(t_{n}\right) \log \bar{R}\left(t_{n}\right)}=0 .
$$

Taking then $t=t_{n}$ in (7.2) and letting $n \rightarrow \infty$, we would get

$$
\lim _{n \rightarrow \infty} \frac{R^{2}\left(t_{n}\right) \log R\left(t_{n}\right)}{t_{n}}=2 A / L
$$

a contradiction.

Theorem 7.2. Let $\theta$ be the radial solution to the Stefan problem (RS) with $n=2$. Then

$$
\lim _{t \rightarrow \infty} \log \mathcal{R}(t)\left|\theta(x, t)-\mathcal{P}_{2}(x, t)\right|=0
$$

uniformly on sets of the form $\left\{x \in \mathbb{R}^{n}:|x| \geq \delta \mathcal{R}(t)\right\}, \delta>0$, where $\mathcal{R}(t)$ is the unique solution for $t>0$ of the equation

$$
\frac{\mathcal{R}^{2}}{2}\left(\log \mathcal{R}-\frac{1}{2}\right)=\frac{A t}{L},
$$

and

$$
\mathcal{P}_{2}(x, t)=\frac{A[\log \mathcal{R}(t)-\log |x|]_{+}}{\log \mathcal{R}(t)} .
$$

Proof. We introduce the rescaled temperature

$$
v(\xi, \tau)=\log \mathcal{R}(t) \theta(\xi \mathcal{R}(t), t), \quad \tau=\int_{0}^{t} \frac{d s}{\mathcal{R}^{2}(s)},
$$


which satisfies

$$
v_{\tau}-\Delta v=\frac{A}{L \log \mathcal{R}(t)}\left(\frac{v}{\log \mathcal{R}(t)}+\nabla v \cdot \xi\right)
$$

if $\alpha(\tau) \leq|\xi| \leq R_{v}(\tau)$, where $\alpha(\tau)=a / \mathcal{R}(t)$ and $R_{v}(\tau)=R(t) / \mathcal{R}(t)$. Moreover,

$$
\begin{array}{ll}
v(\xi, \tau)=0 & \text { if }|\xi|=R_{v}(\tau), \\
v(\xi, \tau)=A \log \mathcal{R}(t) & \text { if }|\xi|=\alpha(\tau) .
\end{array}
$$

We also have that $\lim _{\tau \rightarrow \infty} \alpha(\tau)=0$. On the other hand, Theorem 7.1 implies that

$$
\lim _{\tau \rightarrow \infty} R_{v}(\tau)=1
$$

From here on we can repeat the proof of Theorem 6.5 with minor changes. The most significant ones are that now $w=A[\log d-\log |\xi|]_{+}$and $\psi=\phi / \log \mathcal{R}(t)$. The other ones are obvious.

\section{General solutions to the Stefan problem}

We are now ready to obtain the near and far field limits for general solutions to the Stefan problem, the main purpose of the paper. In both cases the limit coincides with that of the Hele-Shaw flow with the same boundary data.

8.1. Near field limit. We have the following convergence result, exactly like Theorem 4.1 for the Hele-Shaw problem:

Theorem 8.1. Let $n \geq 2$. Then the weak solution of the Stefan problem converges as $t$ tends to infinity to the unique solution, $P$, of the Dirichlet exterior problem (DE) uniformly on compact subsets of $\mathbb{R}^{n} \backslash G$.

We need some auxiliary results.

Lemma 8.2. Let $G_{1}$ be a domain with smooth boundary satisfying $\bar{G} \subset G_{1} \subset \bar{G}_{1} \subset$ $\bar{G} \cup G_{0}$. Then there exists a constant $\delta>0$ such that $\theta(x, t) \geq \delta$ for all $x \in \partial G_{1}$, $t \geq 0$.

Proof. Let $\hat{\theta}$ be the solution to the following initial and boundary value problem:

$$
\begin{cases}\hat{\theta}=\Delta \hat{\theta}, & x \in G_{0}, t>0, \\ \hat{\theta}(x, 0)=h(x), & x \in \Gamma_{0}, \\ \hat{\theta}(x, t)=g(x), & x \in \Gamma, t>0 \\ \hat{\theta}(x, t)=0, & x \in \Gamma_{0}, t>0 .\end{cases}
$$

Then $\theta(x, t) \geq \hat{\theta}(x)$ for $x \in G_{0}, t>0$. The existence of a constant $\delta>0$ such that $\hat{\theta}(x, t) \geq \delta$ for all $x \in \partial G_{1}$ and $t>0$ is a consequence of the strong maximum principle.

We can prove that the support spreads to the whole space. This result was stated without proof in $[\mathrm{M}]$.

Lemma 8.3. The free boundary, $\Gamma(t)$, of a weak solution to the Stefan problem satisfies

$$
\lim _{t \rightarrow \infty} \operatorname{dist}(\Gamma(t), G)=\infty
$$


Proof. We consider a radial solution $\tilde{\theta}$ of (RS) with $a, b, A$ and $\tilde{\theta}(x, 0)$ small enough so that $B_{a} \subseteq G, B_{b} \subseteq \bar{G} \cup G_{0}$, and $\tilde{\theta}(x, t) \leq \delta$ for $x \in \partial G_{1}$, where $\delta$ is the constant provided by Lemma 8.2. Using comparison in $\mathbb{R}^{n} \backslash G_{1}$, we obtain that $\Omega(t) \cup G \supseteq \operatorname{supp} \tilde{\theta}(\cdot, t) \cup B_{a}=B_{R(t)}$, where $R(t)$ is the radius of the free boundary of $\tilde{\theta}$ at time $t$. Theorems 6.4 and 7.1 guarantee that $R(t) \rightarrow \infty$ as $t \rightarrow \infty$, and hence the support of $\theta$ eventually covers every compact subset of $\mathbb{R}^{n} \backslash G$.

We also have an $L^{2}$-estimate for the temperature:

Lemma 8.4. Let $\theta$ be the weak solution to the Stefan problem (WS) for $n \geq 2$. Then

$$
\int_{0}^{\infty} \int_{\mathbb{R}^{n} \backslash G}\left(\frac{\partial \theta}{\partial t}\right)^{2} d x d t \leq C
$$

Proof. We repeat the proof of Lemma 3.4 from [FK], considering penalty functions $\beta_{\varepsilon}$ with the additional property

$$
\beta_{\varepsilon}^{\prime}(t) \leq C / \varepsilon \quad \text { for } 0 \leq t<\varepsilon,
$$

and take into account the remark following Theorem 3 in the same paper.

Proof of Theorem 8.1. We define $\theta_{s}(x, t)=\theta(x, t+s)$. Lemma 6.3 implies that

$$
0 \leq \theta_{s}(x, t) \leq C|x|^{2-n} \text {. }
$$

This estimate and the fact that $\theta_{s}$ is a solution of the heat equation where it is positive provide us with uniform (in $s$ ) interior Hölder estimates for $\theta_{s}, D_{t} \theta_{s}$, $D_{i} \theta_{s}$ and $D_{i j} \theta_{s}$ depending only on $C$ and on the distance of the subset under consideration from the parabolic boundary. Moreover, these estimates are valid up to the fixed boundary $\Gamma \times[0, \infty)$, as this surface is smooth (see $[\mathrm{F}]$ ). The Ascoli-Arzelà theorem guarantees the existence of a sequence $\left\{s_{n}\right\}$ such that $\left\{\theta_{s_{n}}\right\}$ converges to a certain function $\theta_{\infty}(x, t)$ uniformly on compact subsets of $\left(\mathbb{R}^{n} \backslash G\right) \times$ $[0, \infty)$.

The next step is to identify the limit $\theta_{\infty}$. It is straightforward to see that

$$
\begin{cases}\frac{\partial \theta_{\infty}}{\partial t}=\Delta \theta_{\infty} & \text { in }\left(\mathbb{R}^{n} \backslash G\right) \times[0, \infty), \\ \theta_{\infty}(x, t)=g(x) & \text { if } x \in \Gamma\end{cases}
$$

Now,

$$
\begin{aligned}
\int_{\mathbb{R}^{n} \backslash G}|\theta(x, s)-\theta(x, t+s)|^{2} d x & =\int_{\mathbb{R}^{n} \backslash G}\left|\int_{s}^{s+t} \frac{\partial \theta}{\partial t}(x, \tau) d \tau\right|^{2} d x \\
& \leq \int_{\mathbb{R}^{n} \backslash G}\left(t \int_{s}^{s+t}\left|\frac{\partial \theta}{\partial t}(x, \tau)\right|^{2} d \tau\right) d x .
\end{aligned}
$$

Let $K$ be any compact subset of $\mathbb{R}^{n} \backslash G$. The last inequality and Lemma 8.4 tell us that

$$
\lim _{n \rightarrow \infty}\left\|\theta\left(\cdot, s_{n}\right)-\theta\left(\cdot, t+s_{n}\right)\right\|_{L^{2}(K)}=0 .
$$

On the other hand, we know that $\theta\left(x, t+s_{n}\right) \rightarrow \theta_{\infty}(x, t)$ uniformly on $K$, and hence

$$
\lim _{n \rightarrow \infty}\left\|\theta\left(\cdot, s_{n}\right)-\theta\left(\cdot, t+s_{n}\right)\right\|_{L^{2}(K)}=\left\|\theta_{\infty}(\cdot, 0)-\theta_{\infty}(\cdot, t)\right\|_{L^{2}(K)} .
$$

We conclude that $\theta_{\infty}(x, t)=\theta_{\infty}(x, 0)$, i.e., $\theta_{\infty}$ does not depend on $t$. Thus, 


$$
\begin{cases}\Delta \theta_{\infty}(x)=0 & \text { in } \mathbb{R}^{n} \backslash G, \\ \theta_{\infty}(x)=g(x) & \text { if } x \in \Gamma .\end{cases}
$$

Moreover, $0 \leq \theta_{\infty}(x) \leq C|x|^{2-n}$. Thus $\lim _{|x| \rightarrow \infty} \theta_{\infty}(x)=0$ if $n \geq 3$, and $\theta_{\infty}$ is bounded if $n=2$. Therefore $\theta_{\infty}$ is the unique solution of (DE). The uniqueness of the limit implies that convergence is not restricted to a sequence. Hence the result.

8.2. Far field limit. We have the following convergence result, exactly like Theorem 5.1 for the Hele-Shaw problem:

Theorem 8.5. Let $\theta$ be the weak solution to the Stefan problem (WS) and let $C_{*}$, $\mathcal{P}, \mathcal{P}_{2}$ and $\mathcal{R}$ be as in Theorem 5.1.

A) If $n \geq 3$ then

$$
\lim _{t \rightarrow \infty} t^{(n-2) / n}|\theta(x, t)-\mathcal{P}(x, t)|=0
$$

uniformly on sets of the form $\left\{x \in \mathbb{R}^{n}:|x| \geq \delta t^{1 / n}\right\}, \delta>0$.

B) If $n=2$ then

$$
\lim _{t \rightarrow \infty} \log \mathcal{R}(t)\left|\theta(x, t)-\mathcal{P}_{2}(x, t)\right|=0
$$

uniformly on sets of the form $\left\{x \in \mathbb{R}^{n}:|x| \geq \delta \mathcal{R}(t)\right\}, \delta>0$.

Proof. As in the proof of Theorem 5.1, using Theorem 8.1 and Lemma 4.4 we can prove that, given $\varepsilon>0$, there exist values $a$ and $T$, such that, for all $t \geq T$ and $|x|=a$,

$$
\left|\theta(x, t)-\frac{C_{*}}{|x|^{n-2}}\right|<\varepsilon .
$$

Let $m_{+}(t)=\max _{x \in \Gamma(t)}|x|, m_{-}(t)=\min _{x \in \Gamma(t)}|x|$. Let $\theta^{ \pm}$be the solutions to the radial Stefan problem (RS) with boundary data $\theta^{ \pm}=\left(C_{*} / a^{n-2}\right) \pm \varepsilon$ at $|x|=a$, support radii $m_{ \pm}(T)$ at time $t=T$, and continuous and non-negative radial initial data at time $t=T$ such that $\theta^{-}(x, T) \leq \theta(x, T) \leq \theta^{+}(x, T)$. Let $R_{ \pm}(t)$ be the radii of their supports at time $t$. Using comparison for $|x| \geq a, t \geq T$, we get that

$$
\theta^{-}(x, t) \leq \theta(x, t) \leq \theta^{+}(x, t)
$$

for $|x| \geq a, t \geq T$.

A) From (8.1) we get

$$
t^{(n-2) / n} \theta^{-}\left(\xi t^{1 / n}, t\right) \leq t^{(n-2) / n} \theta\left(\xi t^{1 / n}, t\right) \leq t^{(n-2) / n} \theta^{+}\left(\xi t^{1 / n}, t\right)
$$

for $|\xi| \geq a / t^{1 / n}, t \geq T$. But, as $\theta^{ \pm}$are radial solutions, we can apply Theorem 6.5 to obtain that

$$
\lim _{t \rightarrow \infty} t^{(n-2) / n} \theta^{ \pm}\left(\xi t^{1 / n}, t\right)=\left(C_{*} \pm \varepsilon a^{n-2}\right)\left[|\xi|^{2-n}-c_{ \pm}^{2-n}\right]_{+}
$$

uniformly on sets of the form $\{\xi:|\xi| \geq \delta\}, \delta>0$, where

$$
c_{ \pm}=\left(\left(C_{*} \pm \varepsilon a^{n-2}\right) n(n-2) / L\right)^{1 / n}
$$

And from here on the proof continues as in Theorem 5.1.

B) From (8.1) we get

$$
\log \mathcal{R}(t) \theta^{-}(\xi \mathcal{R}(t), t) \leq \log \mathcal{R}(t) \theta(\xi \mathcal{R}(t), t) \leq \log \mathcal{R}(t) \theta^{+}(\xi \mathcal{R}(t), t)
$$

for $|\xi| \geq a / \mathcal{R}(t), t \geq T$. Then we repeat the ideas of $\mathrm{A})$. 
Regarding the free boundary, we can repeat the proof of Theorem 5.2 to obtain that the asymptotic behaviour is exactly that of the Hele-Shaw problem.

Theorem 8.6. Let $m_{+}(t)=\max _{x \in \Gamma(t)}|x|$ and $m_{-}(t)=\min _{x \in \Gamma(t)}|x|$, where $\Gamma(t)$ is the free boundary of the solution of the Stefan problem. Then

$$
\begin{array}{ll}
\lim _{t \rightarrow \infty} \frac{m_{ \pm}(t)}{t^{1 / n}}=\left(C_{*} n(n-2) / L\right)^{1 / n} & \text { if } n \geq 3, \\
\lim _{t \rightarrow \infty} \frac{m_{ \pm}(t)}{(t / \log t)^{1 / 2}}=2\left(C_{*} / L\right)^{1 / 2} & \text { if } n=2 .
\end{array}
$$

\section{ACKNOWLEDGMENTS}

The authors wish to thank the referee for his observations. The work has been partially supported by DGICYT Project PB94-0153.

\section{REFERENCES}

[C] L.A. Caffarelli, The regularity of free boundaries in higher dimensions, Acta Math. 139 (1977), 155-184. MR 56:12601

$[\mathrm{CF}]$ L.A. Caffarelli and A. Friedman, Continuity of the temperature in the Stefan problem, Indiana Univ. Math. J. 28 (1) (1979), 53-70. MR 80i:35104

[D] G. Duvaut, Résolution d'un problème de Stefan (Fusion d'un bloc de glace à zéro degrés), C. R. Acad. Sci. Paris Sér. A 276 (1973), 1461-1463. MR 48:6688

[EJ] C.M. Elliott and V. Janovský, A variational inequality approach to Hele-Shaw flow with a moving boundary, Proc. Royal Soc. Edinburgh 88A (1981), 93-107. MR 82d:76031

[F] A. Friedman, Partial differential equations of parabolic type, Prentice-Hall, Englewood Cliffs, NJ, 1964. MR 31:6062

[FK] A. Friedman and D. Kinderlehrer, A one phase Stefan problem, Indiana Univ. Math. Jour. 24 (11) (1975), 1005-1035. MR 52:6190

[KLV] J.R. King, A.A. Lacey and J.L. Vázquez, Persistence of corners in free boundaries in Hele-Shaw flow, European Jnl. Appl. Math. 6 (1995), 445-490. MR 97a:76037

$[\mathrm{KN}] \quad$ D. Kinderlehrer and L. Nirenberg, The smoothness of the free boundary in the one phase Stefan problem, Comm. Pure Appl. Math. 31 (1978), 257-282. MR 82b:35152

[L] A.A. Lacey, Bounds on solutions of one-phase Stefan problems, European Jour. Appl. Math. 6 (1995), 509-516. MR 96j:80009

[LR] B. Louro and J.F. Rodrigues, Remarks on the quasi-steady one phase Stefan problem, Proc. Royal Soc. Edinburgh 102A (1986), 263-275. MR 88e:35186

[M] H. Matano, Asymptotic behavior of the free boundaries arising in one phase Stefan problems in multi-dimensional spaces, in "Nonlinear Partial Differential Equations in Applied Science" (Tokyo, 1982), North-Holland Math. Stud., 81, 1983, pp. 133-151. MR 86a:35151

[Me] A.M. Meirmanov, The Stefan problem, Walter de Gruyter, Berlin, 1992. MR 92m:35282

[MGR] J.A. McGeough and H. Rasmussen, On the derivation of the quasi-steady model in electromechanical machining, J. Inst. Math. Applics. 13 (1974), 13-21.

[R] S. Richardson, Hele-Shaw flows with a free boundary produced by the injection of fluid into a narrow channel, J. Fluid Mech. 56 (1972), 609-618.

[Ru] L.I. Rubinstein, The Stefan problem, Transl. Math. Monographs, vol. 27, Amer. Math. Soc., Providence, RI, 1971. MR 50:3837

[ST] P.G. Saffman and G.I. Taylor, The penetration of fluid into a porous medium Hele-Shaw cell containing a more viscous liquid, Proc. Royal Soc. London Ser. A 245 (1958), 312329. MR 20:3697

[V] J.L. Vázquez, Singular solutions and asymptotic behaviour of nonlinear parabolic equations, in "International Conference on Differential Equations; Barcelona 91" (Equadiff91), (C. Perelló, C. Simó and J. Solà-Morales eds.), World Scientific, Singapore, 1993, pp. 234-249. MR 95a:35067

Departamento de Matemáticas, Universidad Autónoma, 28049 Madrid, Spain

Departamento de Matemáticas, Universidad Autónoma, 28049 Madrid, Spain 